\title{
El mesianismo político de Augusto Pinochet y la lucha por el espacio sacrificial $^{1}$
}

\author{
Pablo Ortúzar Madrid ${ }^{2}$ \\ Carolina Tomic López ${ }^{3}$ \\ Sebastián Huneeus Valenzuela ${ }^{4}$
}

\begin{abstract}
Resumen
Se analiza la dimensión simbólica de la figura de Augusto Pinochet y su manifestación religiosa en el pinochetismo. Se explica esta manifestación a partir de la confluencia de tres factores profundamente arraigados en la cultura latinoamericana: la hacienda, la religiosidad popular y el sacrificio. Se explica cómo el pinochetismo sitúa a Pinochet en el lugar del cordero sacrificial y como padre salvador y protector de la "familia chilena", y cómo se desarrolla este mito en oposición a la izquierda, que intenta leer la historia del país desde el sacrificio de Allende. Se concluye que la disputa por el espacio sacrificial imposibilita simbólicamente la reconciliación nacional; en efecto, la Concertación acepta el "nuevo orden pinochetista" (post-dictadura) a la vez que se funda en su crítica.
\end{abstract}

Palabras clave: Reconciliación política, Pinochet y Allende, cultura y política

\begin{abstract}
We analyze the symbolic dimension of the figure of Augusto Pinochet and the manifestation of religion in Pinochet. It explains the event from the confluence of three factors deeply rooted in Latin American culture: the farm, popular piety and sacrifice. It explains how the Pinochet puts in place the sacrificial lamb as a father and savior and protector of the "Chilean family," and how this myth developed in opposition to the left, which tries to read the history from the slaughter of Allende. We conclude that the dispute over the sacrificial space symbolically national reconciliation impossible, in fact, the Coalition accepts the "new order Pinochet" (post-dictatorship) while it is based on his criticism.
\end{abstract}

Keywords: Political reconciliation, Pinochet and Allende, Culture and politics.

1 Monografía ganadora de la $1^{a}$ Mención de Honor en el Concurso de monografías sobre Religiosidad Popular en América Latina versión 2007, convocado por la Asociación de Cientistas Sociales de la Religión en el MERCOSUR (ACSRM) y presentada en las XIV Jornadas sobre Alternativas Religiosas en América Latina realizada en la Universidad Nacional de San Martín, Buenos Aires, en septiembre del 2007.

2 pablo.ortuzar.madrid@gmail.com

3 topatomic@gmail.com

4 sebastete@yahoo.es 
El mesianismo político de Augusto Pinochet y la lucha por el espacio sacrificial - Pablo Ortúzar Madrid, Carolina Tomic López, Sebastián Huneeus Valenzuela

\section{Presentación}

"Quiero señalar que acepto esta nueva cruz, con la humildad del cristiano y el temple de un soldado, si con ello presto un servicio a Chile y a los chilenos ${ }^{\prime \prime}$.

El conflicto ideológico-político que hasta el día de hoy representa Augusto Pinochet Ugarte en Chile quedó evidenciado con las recientes manifestaciones masivas producidas a causa de su fallecimiento. Miles de personas en duelo frente al Hospital Militar y miles de personas celebrando en la céntrica Plaza Italia, todas ellas reunidas y separadas por una persona en común. Este hecho sorprendió a aquellos que pensaban que en Chile las diferencias y heridas que quedaron desde el régimen dictatorial de Pinochet eran ya cosa del pasado.

Esta situación nos presenta una disyuntiva relevante para nuestra sociedad y para todas aquellas sociedades latinoamericanas que también vivieron regímenes dictatoriales semejantes, lo cual nos incentiva a investigar y reflexionar. Se hace necesario indagar sobre las bases de la legitimidad de Pinochet y preguntarnos cómo y por qué éste es capaz de convocar tales actitudes y conductas -bastante propias del fanatismo religioso-, las cuales creemos que, en determinados grupos sociales, llegan a sustentarse en expresiones de una religiosidad popular, que si no fue originada, al menos fue potenciada desde el discurso público y el manejo mediático (quizás inconsciente pero efectivo). Ello dado que la legitimidad o permanencia de un líder puede depender más de su capacidad carismática y manipuladora que de las promesas realmente cumplidas.

Con ese objeto, hemos de enfocarnos heurísticamente en uno de esos dos grupos de manifestantes: en aquel que, viviendo el duelo, fue capaz de expresar un fanatismo e idolatría insospechados. Y de acuerdo a lo anterior, penetraremos en la base cultural

5 Augusto Pinochet, citado en Lagos Schuffeneger, H. (2001), p. 51. 
profunda que une y separa a estos dos grupos, en donde nuestra matriz cristiana, nuestro pasado hacendal y la estructura del mecanismo sacrificial tienen mucho que decir.

\section{Religiosidad popular y su manifestación en Chile}

“Ustedes saben que el pueblo chileno oraba por su salvación y que hoy se siente libre y apartado del mal"6.

\section{Concepto y alcance de la religiosidad popular}

Entenderemos el concepto de religiosidad popular como la realización concreta de una religión. No es una religión nueva institucionalizada, sino creencias particulares que se integran dentro del sustrato cultural común de una sociedad. La religiosidad popular se constituye como tal a través de una dialéctica respecto del "culto oficial" y dentro de una determinada estructura sociocultural. En la búsqueda de expresiones de religiosidad popular ligadas al pinochetismo, no deben considerarse solamente las distinciones socioeconómicas, sino mayormente el grado de adscripción al pinochetismo en cuanto proyecto de sociedad.

Sociológicamente, "las condiciones que dan cuenta de la existencia de la religiosidad popular pueden ser reducidas a las siguientes: la existencia de una doctrina religiosa reconocida como 'oficial'; la presencia de un grupo de especialistas reconocidos como 'decodificadores oficiales' de estas doctrinas -los que en la práctica se transforman en sus fuentes terrenas-; una heterogeneidad social que implique una distribución diferencial de los conocimientos al interior de un sistema social. (...) la religiosidad popular inexorablemente se debe, nutre y opone a una forma religiosa oficial" (Arnold, Skewes, Prado, 1984, p. 3).

La religión se plantea como idea-fuerza capaz de motivar y reglamentar conductas de acuerdo a la capacidad de influencia

6 Augusto Pinochet, citado en Lagos Schuffeneger, H. (2001), p. 22. 
del líder carismático que la pregona. Por esto el vínculo entre la religiosidad popular y la dominación política es fundamental para nuestra reflexión.

\section{Religiosidad popular en Chile}

En Chile "no se puede hablar de Religiosidad Popular con un padrón único" (Aliaga, 1992, p. 25). En el caso de la zona central de Chile, encontramos un escenario de fuerte absorción del elemento religioso indígena por parte de los evangelizadores cristianos. El espacio de sometimiento e integración de éstos fue el de la encomienda y la merced de tierras. Otra institución que se formó con el correr de los siglos fue la hacienda, la cual puede considerarse como un "centro donde se plasman las prácticas de la religiosidad popular campesina, la cual mantuvo su ancestro indígena" (Ibíd., p. 29).

La religiosidad popular chilena se vio influenciada por las distintas formas de relacionarse que tuvo el indígena con el conquistador, luego con el criollo y después con el mestizo chileno que de ambos nació. Estas relaciones de trabajo forzado entre el encomendero y sus indios, o aquellas totalmente paternalistas entre el patrón y sus peones e inquilinos (indígenas sin trabajo o criollos empobrecidos), dieron lugar a rituales y tradiciones, creencias y prácticas que fueron transmitiéndose por generaciones y que hoy se mantienen manifiestas en nuestra estructura cultural profunda.

Para explicarnos la síntesis tras la superposición y absorción religiosa del catolicismo de Castilla de las religiones indígenas de América, utilizamos a Pedro Morandé (1980). Para Morandé, la hacienda es la expresión más pura del sincretismo religioso dado en Latinoamérica, es decir, de su religiosidad popular. Esta institución económico-administrativa y social mantiene su régimen hasta inicios del siglo XX y, por tanto, su influencia hasta nuestros días, dada por las figuras que de ella se desprenden, como 
la del patrón-padre. Estas figuras, o sujetos históricos, nos sirven para entender la clase de vínculo que actualmente mantienen los líderes y autoridades políticas con el pueblo.

Para Pedro Morandé, en la hacienda confluyen el cristianismo, que es una religión de palabra, es decir, se basa en la eficacia de la fe para asegurar la reconciliación del hombre con Dios, y las religiones indígenas y negras, que son de carácter cúltico, definiendo sus relaciones con las divinidades a través de la eficacia simbólica de los rituales. Aceptando que el catolicismo se asume como religión de la palabra revelada, tiene, entre sus formas de expresión de la palabra, rituales (como la eucaristía y los sacramentos) que por lo mismo pueden y son recibidos por las religiones cúlticas nativas en forma natural. Por otro lado, lo cúltico viene dado por la cotidianidad, por ser parte de la vida doméstica y diaria, sin separación de la religión con la vida social y natural, situación que también permite, por tanto, el surgimiento de nuevas formas de expresiones religiosas.

La cuestión de la hacienda es central para comprender los procesos culturales que afectan a la sociedad chilena actual, ya que en algún lugar de nuestras memorias, narrativas, identidades y estructuras normativas, la sigilosa influencia de la matriz hacendal se hace ineludible. Es por esto que la retomamos no solamente en cuanto estructura básica de socialización y configuración histórica, sino para comprender los alcances de un pasado que probablemente se actualiza en la adoración a una figura salvacionista y paternal como lo fue Augusto Pinochet.

Pero el meollo del asunto sigue siendo más complejo todavía. Para resolverlo, esperamos que a través de la teoría de la crisis mimética y del mecanismo sacrificial de Girard, podamos comprender mejor la afinidad entre la estructura de nuestra religiosidad popular con el gobierno militar. 


\section{Realidad y función del sacrificio}

“Ojalá el mío fuera el último sacrificio (...). Quienes creemos en el perdón y en la reconciliación verdadera tenemos que seguir trabajando duramente por el futuro (...). Soy absolutamente inocente de todos los crímenes y de los hechos que irracionalmente se me imputan (...). Conservo intacta mi fe en Dios y en los principios que han guiado mi existencia"7.

\section{Crisis mimética y sacrificio}

René Girard (1982; 1984) plantea que para comprender la cultura debemos penetrar en el conflicto mimético, que surge del encuentro de voluntades en el mismo objeto, que provoca una crisis de mimesis que se propaga con mortal facilidad. El principio fundamental de esta mimesis radica en la naturaleza particular del deseo, el cual elige sus objetos gracias a la mediación de un modelo. En esta línea, la dimensión adquisitiva de la mimesis es además su dimensión conflictiva, al derivar en el mimetismo de apropiación. Esta violencia desatada, una vez olvidado el objeto de deseo, se llama mimesis del antagonista. En contrapartida, la represión cultural de la mimesis de apropiación construye el entredicho, la norma prohibidora de la violencia. Así, en Girard la cultura nace como un intento de normar la violencia recíproca producida por la mimesis de apropiación.

Las comunidades arcaicas entraban en crisis violentas que se resolvían al descubrir y expulsar o eliminar al supuesto causante de la violencia. La cohesión de la comunidad se lograba entonces gracias al sacrificio de una víctima arbitraria, muchas veces inocente, que se volvía el chivo expiatorio, pasando por responsable de toda la crisis mimética. A partir de esto, Girard llega a la comprensión de la génesis de lo humano a través de la crisis resuelta por el mecanismo de la víctima propiciatoria. Tanto la violencia recordada como el fin de la misma quedan conservados -aunque

7 Augusto Pinochet, citado en Lagos Schuffeneger, H. (2001), pp. 51-52. 
velados- bajo ritos, mitos y entredichos, que nos previenen, a través de la simulación, de volver a enfrentar una crisis mimética. Sin embargo, apenas se ve resquebrajado el orden logrado y dichas instancias pierden eficacia simbólica, vuelve a surgir la violencia y la crisis, lo que sólo se arregla con un nuevo sacrificio.

Si esa víctima, viviente en la comunidad, le traía la muerte, y una vez muerta, le trae la vida, habrá que concluir inevitablemente que su aptitud para trascender los límites de la humanidad ordinaria tanto en el mal como en el bien se extiende a la vida y a la muerte. Si hay para ella una vida que es muerte y una muerte que es vida, es que las fatalidades de la condición humana no afectan a lo sagrado. Se esbozan así todos los rasgos de la trascendencia religiosa. La víctima se vuelve prodigiosamente significante y específica. En ella se da el paso de lo aleatorio a lo específico y la vuelta a lo diferenciado. Es una fuente rica de significaciones.

Luego, argumenta Girard, surge la jerarquía social a partir de la voluntad de reproducir el mecanismo reconciliador. Se busca una nueva víctima que se parezca a la idea que se tiene de la primera. $Y$ en este nuevo sujeto se descargan ambos momentos sacrificiales: el odio y la alabanza. Esta víctima, rey de los hombres, aparece gracias a ambas transferencias, heredando de la víctima original un prestigio sobrehumano y terrorífico. La víctima-rey, por su parte, puede ir aplazando su sacrificio hasta el infinito, haciendo dócil al pueblo, ofreciendo sacrificios sustitutivos del suyo propio y legando la posición de víctima a su hijo, y así. Pero si su soberanía no se cristaliza en un poder concreto, lo más seguro es el regreso del sacrificio real. Así, Girard plantea que existe un vínculo simbólico entre la soberanía y el sacrificio.

\section{Sacrificio y legitimación del poder}

Hinkelammert (2003) plantea que los asesinatos fundantes son constructos, pero que ninguno es fundante por sí solo. Sin embargo, éstos forman parte de la constitución del poder, pues a través 
de ellos logra legitimarse frente a los dominados, como frente a quienes lo ejercen. De este modo, el liquidado puede ser un héroe inocente, y en este caso, el poder se percibe como reencarnación del héroe frente a sus asesinos, enemigos del poder. No obstante, el asesinado puede ser de igual manera un héroe negativo y, por consiguiente, un culpable matado legítimamente. Entonces el poder se percibe como reencarnación de estos asesinos y a sus enemigos como reencarnación del "demonio" asesinado. Los enemigos del poder son tratados entonces como encarnación de los que cometieron el asesinato fundante. El poder se presenta ahora como vengador de un asesinato fundante frente a los asesinos que lo cometieron y la comunidad se divide. El grupo de vengadores es un grupo de asesinos; con todo, su asesinato es una venganza.

El magnicidio se entiende como el homicidio del padre de todos los hombres del reino. A partir de esto, en la persecución de los enemigos del poder aparece constantemente su denuncia como matadores del padre. La figura sagrada asesinada funda el poder, e incluso el imperio, a través de la legitimación de la persecución de sus enemigos. Éstos, quienes son asesinados en nombre de la retribución por el asesinato fundante, son denigrados en términos absolutos y pierden su dignidad.

En la tradición griega muy pronto apareció la identificación del rey padre con la ley padre y, por ende, la autoridad era la autoridad de la ley. Luego de la imperialización del cristianismo, la crucifixión de Jesús fue entendida como el asesinato de Dios, rey, padre y ley. Los enemigos del imperio ya no son apenas asesinos del rey y padre, sino asesinos de Dios, rey y padre. Así, la reivindicación del poder es ya universal y los enemigos del poder son ahora aliados del enemigo universal de Dios, es decir, del diablo. Por lo tanto, cualquier cuestionamiento al imperio y al cristianismo imperializado -la ortodoxia cristiana- es levantarse contra Dios y su ley. Pero como tal, es participación en la crucifixión de Cristo. Es, paradójicamente, estar al servicio del diablo. 
Este mecanismo se fundamenta en la legitimación del poder y funciona gracias a que la construcción de pretextos de asesinato opera por medio de la anticipación de horrores futuros imaginarios: todo es lícito y los propios Derechos Humanos desaparecen. Respetarlos produciría tal horror que el crimen resulta ser el mismo respeto a los derechos humanos.

\section{La dialéctica sacrificial chilena}

Para el pinochetismo, el 11 de septiembre de 1973 representa el advenimiento de un salvador vengativo: Augusto Pinochet. El asesinato fundante que reclama venganza es, dentro de esta lógica, el del orden y la institucionalidad, el de la ley, perpetrado por el gobierno socialista de Salvador Allende ${ }^{8}$. Desde esta perspectiva, al ser la ley la que pretende distinguir, ordenar y clasificar los hechos sociales, el país se encontraba sumido en una crisis mimética. La ley, para un sector de nuestra sociedad que cree en el derecho natural, es, además, expresión viva de la voluntad de Dios Padre en nuestra patria. Por lo tanto, además, los marxistas chilenos no son otra cosa que la expresión política de una voluntad demoníaca; de ese modo, es Dios Padre quien, viendo la destrucción de su obra, envía al general Pinochet a traer el nuevo orden que deberá instituirse sobre las ruinas del anterior. Es el "patrón", padre hacendal, en cuyo cobijo cabe toda la "gran familia chilena". De hecho, como señala el pinochetismo, se trata de un nuevo padre de la patria. Pero para instaurar el nuevo orden y abrir a los hijos de Chile este nuevo reino de alegría y prosperidad, deberá cargar con la cruz para ser finalmente sacrificado. Y su calvario comienza aquel mismo 11 de septiembre, cuando se ve obligado a empuñar la espada contra su propio gobierno, rompiendo una supuestamente larga e intachable tradición institucional del ejército chileno. Y continúa cuando debe comandar la desaparición y muerte de aquellos contaminados por Satán, sabiendo que la venganza, tarde o temprano, llegará y que la ex-

8 Recuérdese que antes del golpe, el gobierno de la Unidad Popular fue acusado por el centro y la derecha de inconstitucionalidad. 
piación final será sobre su propia muerte. Así, el pinochetismo cree lograr justificar la persecución, muerte y secuestro de muchos ciudadanos como prevención de una crisis superior'.

Para el pinochetismo religioso, Pinochet se configura como un hijo de Dios, Cristo redentor. Esto coincide con el hecho de que en el discurso religioso del dictador son muy pocas las referencias a Cristo, apelando éste más comúnmente a la Virgen del Carmen y a Dios, de quien implícitamente se arroga el poder de ser el intérprete en la Tierra. Esta idea se ve reforzada por el relato de una de las anécdotas más potentes que guarda el pinochetismo: el atentado que sufrió la caravana de Pinochet el año 1986, realizado por el Frente Patriótico Manuel Rodríguez, de donde salió ileso, según su relato, por la intervención de la Virgen del Carmen, santa patrona del ejército chileno, cuya imagen habría visto el dictador en los cristales trizados por la descarga de bazuca que se suponía terminaría con su vida.

Por su parte, la izquierda chilena también ha construido su mito desde el discurso victimal, comprendiendo a Allende como el Cristo redentor, quien se habría sacrificado por los pobres, por los oprimidos, al igual que lo hiciera Jesús, y también por el orden y la institucionalidad republicana y sus valores fundantes ${ }^{10}$.

Leyendo esta dialéctica, en la cual cada uno de los participantes intenta restaurar el orden patrio a partir de la construcción de un discurso desde los sacrificados, acusando al otro bando de ser los sacrificadores, resulta interesante notar el hecho de que el primer Jesús (Pinochet) se acomoda a la imagen del "Cristo emperador", mientras que el segundo (Allende) es el "compañero Cristo" ("compañero presidente", le llamaban), siendo el primero hijo de Dios en el sentido estricto, y el segundo, "hijo entre

9 Se habla incluso, en la época del golpe, de un supuesto plan de la izquierda para asesinar a toda la oposición al allendismo: El Plan Z.

$10 \mathrm{Al}$ negarse a salir de La Moneda, muere defendiendo la dignidad del cargo y la institucionalidad del país frente a los militares que "han traicionado el juramento a su bandera". 
hijos", hermano, y apelando, por tanto, a dos visiones teológicas divergentes de la imagen de Cristo.

Interesante resulta, además, notar que en nuestro país esta dialéctica no ha encontrado solución y que el discurso de la "reconciliación nacional" implementado por la Concertación y la Alianza por Chile ha sido simplemente vano, por basarse en la idea de dejar atrás las heridas del pasado. Es más, la estrategia de gobierno de la Concertación ha consistido en gobernar desde la posición sacrificial, proyectándose a sí misma como cargadora de la cruz neoliberal heredada de la dictadura, debiendo exponerse, por tanto, al sacrificio de replicar las lógicas políticas de los crucificadores, por quienes fueran torturados ${ }^{11}$, además de traicionar a los pobres del país. Por el contrario, la oposición de la Alianza por Chile ha insistido en que la cruz neoliberal que actualmente carga la Concertación no es más que la respuesta necesaria a la crisis mimética acaecida durante la Unidad Popular, en donde el riesgo de violencia social e indiferenciación era tal, que el asesinato de la república, para volverla a dar a luz, fue inevitable.

\section{Pinochet, su discurso y la "reconciliación nacional"}

"Más allá de mis dolores y de las heridas que llevo en el alma por las injustas vejaciones de que he sido objeto (...) quiero señalar que acepto esta nueva cruz, con la humildad del cristiano y el temple de un soldado, si con ello presto un servicio a Chile y a los chilenos ${ }^{\prime \prime 2}$.

11 Hay que considerar la importancia que tuvo en las últimas elecciones presidenciales el hecho de que Michelle Bachelet, la actual presidenta de Chile, hubiera sido torturada en Villa Grimaldi por efectivos de la CNI y que, sin embargo, superando este hecho, perdonara y hablara de reconciliación.

12 Augusto Pinochet, Carta de navidad. Citado en Lagos Schuffeneger (2001), p. 51. 


\section{El discurso público de Pinochet}

Según Munizaga (1983), el derrocamiento de la Unidad Popular vació sustantivamente las narrativas y sentidos de buena parte de la sociedad chilena, lo cual fue instrumentalizado por el discurso público de Pinochet para obtener legitimidad, especialmente durante los primeros años de dictadura (1973-1976). Fue en este contexto caótico y violento donde el discurso de Pinochet logró erigirse como el único con la posibilidad de referirse a la "totalidad" de los chilenos públicamente. Según Munizaga, dicho discurso tuvo la cualidad de estar motivado hacia la consolidación de un nuevo marco de significaciones sobre el cual los sujetos pudieran orientar sus vidas normalmente.

En particular, la relevancia del discurso de Pinochet en ese momento histórico concreto estribó en que presentó un nuevo proyecto de sociedad nacional basado en una relectura particular de la historia y expresado en determinadas configuraciones simbólicas. Y cabe decir que el destinatario del discurso era la "gran familia chilena", dentro de la cual el ejército había de cumplir una función paternal y moral, legitimada por su carácter arquetípico conferido por el nuevo discurso histórico. Se desprende que el "gran padre" de la "familia chilena" es Pinochet, el cual se ve obligado a sacrificarse durante los diecisiete años de dictadura, para evitar la violencia social y una nueva violación a la patria. Dentro de esta "gran familia chilena", cada integrante tiene el deber de cumplir con la función que le ha sido asignada; "ser lo que son y hacer lo que hacen cotidianamente. (...) acatamiento del poder en cuanto encarna y conduce al orden perfecto" (Munizaga, Ochsenius, 1983, p. 73). En efecto, según este análisis, el gobierno militar fue la restauración de la pax hacendal, del gran orden jerárquico y tradicional que por siglos gobernó a Chile. Pero las bases sociales de la legitimidad radicaron con mayor profundidad en el carácter mesiánico de Pinochet. 


\section{El general Pinochet y el mesianismo político}

Lagos Schuffeneger, en El general Pinochet y el mesianismo político (2001), analiza el régimen militar desde el punto de vista del empleo de elementos religiosos para su legitimación. Plantea que su estrategia transcurrió doblemente por la vía del mesianismo, como la cruzada ideológica para derrocar al enemigo, y en el orden sacrificial, como lo demuestran el atentado en 1986, su detención en Londres en 1998 y su tono discursivo hacia la "gran familia chilena". A nuestro juicio, es aquí donde nos acercamos decisivamente a un espacio que podríamos entender como una religiosidad popular ligada al pinochetismo, pues un régimen que busca legitimarse religiosamente debe encontrar una base social que así lo permita.

El primer recurso al que se refiere Lagos es el de la Guerra Santa, la dictadura como una cruzada de la fe entre marxistas ateos y pinochetistas creyentes. Con esto fue satanizado el socialismo. Consiguientemente, se desprende que el golpe de Estado se constituyó como una "respuesta divina" a las fauces del infierno que amenazaron a nuestra patria. De hecho, en 1977 y en palabras del mismo Pinochet: "Ustedes deben saber que el movimiento del 11 de septiembre fue especialmente dirigido a salvar la parte espiritual del pais"13.

\section{Legitimidad y sacrificio}

Pinochet, durante la dictadura, se hizo leer en clave victimal por el pueblo chileno, como un chivo expiatorio y enviado de Dios frente a la crisis social que tuvo lugar durante la Unidad Popular. Por lo tanto, en su discurso estaba ineludiblemente atado a tiranizar a la izquierda y al marxismo, pues perder de vista al enemigo ideológico es en el fondo perder el fundamento mediante el cual funciona la legitimidad cuando está inscrita dentro de la lógica sacrificial.

13 Augusto Pinochet, citado en Lagos Schuffeneger, H. (2001), p. 23. 
En vista de que el sacrificio es una rica fuente de legitimidad para catalizar la paz social, el discurso del general durante la dictadura adquirió un matiz de sufrimiento vicario. Posteriormente, la detención en Londres y el escándalo del Riggs Bank fueron el calvario final del general. Fue desde Londres que en diciembre de 1998 el senador envió a todos los chilenos su "Carta de Navidad", donde expresaba su deseo de ser él la víctima, asumir todas las culpas a pesar de ser inocente y legar a Chile el orden y la paz. Es Cristo que avanza cargando la cruz hacia su sacrificio final. Y en su camino se encuentra con los apóstoles del pinochetismo, que limpian sus heridas, viajan a Londres y protestan en los medios de comunicación. Pero luego Pinochet fue acusado de enriquecimiento ilícito a costa del Estado chileno, al descubrirse en Miami diversas cuentas bancarias de su propiedad abiertas con nombres falsos. Se hablaba de cifras millonarias. Cristo es puesto en la cruz entre ladrones. Está desnudo y su costado sangra. Pinochet es un cadáver político, un muerto en vida. Sus partidarios más fervientes, sus funcionarios, la derecha chilena, lo ignoraron una y mil veces. Los candidatos presidenciales de la derecha se disputaron la lejanía con el general. Y finalmente no se logró demostrar que esos dineros hayan sido robados y sí que las cifras habían sido exageradas. Posteriormente, el gobierno concertacionista acusó a Pinochet, en base a un mail de dudosa procedencia, de tener millones de dólares en lingotes de oro en un banco en Asia. La acusación fue burda. Un manto de dudas se tejió sobre el resto de las acusaciones económicas que pesaban sobre el general. $\mathrm{Y}$ luego, Pinochet falleció, y en su muerte material resucita espiritualmente para el pinochetismo. En la Plaza Italia se celebró con champaña. En el Hospital Militar se cernió un manto de dolor. Y se manifestó el abismo que divide a Chile. No hubo honores de Estado para su funeral, pero sí máximos honores militares. Treinta mil personas lo despidieron en medio de banderas chilenas. El general sube a los cielos y su mito se inscribe en los corazones de sus partidarios. 


\section{La reconciliación sin tumbas}

Según Girard, la cultura se elabora siempre como tumba, la cual no es más que el primer monumento humano que hay que elevar en torno a la víctima expiatoria. Pero nuestro Chile actual rebosa de muertos sin sepultura. Los detenidos desaparecidos y los militares y policías muertos yacen en la intemperie republicana. Los cadáveres de Allende y los detenidos desaparecidos yacen junto a los primeros. ¿A quién declarar víctima sacrificial? ¿Sobre quién erigir este primer monumento humano? ¿Quiénes son las víctimas? ¿Quiénes los victimarios? El tema no es menor, pues a partir de la sacralización de la imagen de uno de estos chivos expiatorios se legitimará el orden político que esté por venir, reclamándose el bando interesado como sustituto de la víctima-rey y resignificando nuestra historia y cotidianidad a partir de la imagen de la víctima. He aquí lo central del problema de la lucha por la llamada "memoria histórica". Nuestra actualidad política es incapaz de responder estas preguntas. La coalición de gobierno de las víctimas de la dictadura (la Concertación) conduce y alaba el "nuevo orden" traído por el régimen militar, como justificando su necesidad histórica, a la vez que funda su identidad en la negación de éste. Esto provoca que nuestra cultura política sea una fosa común, poblada de víctimas anónimas, sacrificadas en nombre de la patria.

\section{Conclusiones}

Hemos de mencionar la necesidad del pensamiento dialéctico para analizar y comprender ciertos procesos históricos y culturales. En nuestra reflexión, éste se hizo necesario a la hora de emplear un concepto de religiosidad popular, tras lo cual llegamos a que ésta se constituye como tal dialogando con la tradición religiosa "culta", obviando analíticamente las diferencias de clase. Asimismo, la lucha por el espacio sacrificial se logra aprehender si se piensa en términos de la dialéctica que se establece entre dos 
grupos disputándose simbólicamente la posición de chivo expiatorio, para salvaguardar la legitimidad de su poder.

En estos términos, hemos logrado comprender y reconstruir el discurso mítico del pinochetismo y explicar desde aquél los procesos históricos relativos a la dictadura militar de Pinochet, su proceso posterior y la legitimidad de que gozó y goza en una gran parte de la población chilena. En este discurso, Pinochet se reconoce a sí mismo como el Cristo redentor y se comprende el proceso que lleva desde el golpe de Estado a la muerte de Pinochet como un largo vía crucis, un sacrificio necesario.

Consideramos, además, que el discurso del pinochetismo proyecta una expresión de religiosidad popular en la medida en que los sucesos políticos son leídos por éste en clave religiosa, idea que se ve confirmada en los gestos espontáneos de algunos de sus partidarios luego de su muerte, que reclamaban su santidad y le adjudicaban milagros. Este tipo de manifestaciones probablemente se seguirán dando en el tiempo, ya que las condiciones de posibilidad para que fenómenos como éste se lleguen a concretar están absolutamente dadas ${ }^{14}$.

Por otra parte, consideramos que la imagen de Pinochet se inscribe en la lógica del patrón de hacienda, del pater, estructura profundamente arraigada en nuestra cultura y que, desde la visión pinochetista, es una respuesta simbólica al ausentismo de este padre durante el período pre-golpe. Esto explicaría, además, la carga religiosa de la imagen de Pinochet, ya que el orden hacendal se basaba justamente en un orden religioso, estando significados de esta forma todos sus actores, y en especial, el patrón.

Respecto de nuestro Chile actual, postulamos que la dificultad de reconciliación dentro del país está mediada por el intento de los dos grandes bloques de poder (la Concertación y la Alian-

14 Cabe mencionar el caso de la espiritista Verónica Pantich, quien, tras la defunción del ex general, dijera en una entrevista que "Pinochet será santo y pronto hará milagros" (diario Las Últimas Noticias, Santiago de Chile, 26 de diciembre de 2006). 
za por Chile) de reconstruir su historia desde una posición de víctima, poniendo unos a Allende como cordero sacrificial y los otros a Pinochet. Esto hace muy difícil la síntesis reconciliatoria y provoca que se siga gobernando desde el mecanismo victimal.

Por último, consideramos que a partir de nuestra investigación obtuvimos un interesante marco comprensivo para la interpretación de la historia latinoamericana, en particular de sus procesos políticos y su relación con nuestra cultura religiosa, en vista de las similitudes que tenemos con otros países de la región.

\section{Bibliografía}

Aliaga Rojas, Fernando (1992). Religiosidad popular chilena: visión histórica. Ediciones Paulinas, Santiago de Chile.

Arnold, Marcelo; Skewes, J. Carlos; Prado, M. Teresa (1984). Expresiones comunitarias de la religiosidad popular en Chile: sugerencias metodológicas e interpretativas. Universidad de Chile, Departamento de Antropología, Santiago de Chile,

Girard, René (1984). Literatura, mimesis y antropología. Gedisa, Barcelona. (1982). El misterio de nuestro mundo. Claves para una interpretación antropológica. Sígueme, Salamanca.

Hinkelammert, Franz (2003). El asalto al Poder Mundial y la violencia sagrada del Imperio. DEI, San José de Costa Rica.

Lagos Schuffeneger, Humberto (2001). El general Pinochet y el mesianismo político. $1^{\text {a }}$ ed., LOM Ediciones, Santiago de Chile.

Morandé, Pedro (1980). Ritual y palabra: Aproximación a la religiosidad popular latinoamericana. Centro Andino de Historia, Lima.

Munizaga, Giselle; Ochsenius, Carlos (1983). El discurso público de Pinochet: (1973-1976), precedido por el ensayo "Políticas de comunicación bajo regímenes autoritarios: el caso de Chile". $1^{a}$ ed., CLACSO, Buenos Aires. 